\title{
An Adaptive Spectrum Detection Mechanism for Cognitive Radio Networks in Dynamic Traffic Environments
}

\author{
Shensheng Tang and Brian L. Mark \\ Dept. of Electrical and Computer Engineering \\ George Mason University, Fairfax, VA
}

\begin{abstract}
We propose an adaptive spectrum detection mechanism for cognitive radios in a dynamic traffic environment. Cognitive radios generate secondary calls, which opportunistically make use of channels left idle by primary traffic generated by the licensed radios in the system. Spectrum detection for the cognitive radios is formulated as a hypothesis testing problem based on the Bayes criterion to minimize average cost. The maximum likelihood estimates of the prior probabilities for the hypothesis test are obtained from the dynamics of both traffic types of traffic using a Markovian model of the system channel occupancy. The spectrum detection scheme is extended to incorporate cooperation among multiple secondary users. Our numerical results suggest that the adaptive spectrum detection scheme can achieve significantly better error performance than a static scheme that ignores the prior probabilities, especially under light primary traffic conditions. Cooperative spectrum detection among multiple users can further reduce the false alarm and misdetection probabilities.
\end{abstract}

\section{INTRODUCTION}

Cognitive radios have been proposed as the means to promote the efficient use of the spectrum by exploiting the existence of spectrum holes (i.e., unused spectrum) [1], [2]. In cognitive radio (CR) networks, users equipped with CRs are referred to as secondary users, who opportunistically share the spectrum resources with the licensed users of the existing system. The licensed users are referred to as primary users. By allowing secondary users to reclaim idle channels, a much higher spectrum efficiency can be achieved [3].

Recent research on cognitive radio networks has been done using statistical signal processing, e.g., [4]-[6], and queuing theory, e.g., [3], [7], as tools for design and analysis. In [4], a measurement-based model was proposed to statistically describe the busy and idle periods of a WLAN. Two different sensing strategies, energy-based detection and feature-based detection, were explored to identify spectrum opportunities. In [5], a sensing-based approach was studied for channel selection in spectrum-agile communication systems. In [6], a constrained optimization method was proposed to estimate the transmission power and position of the primary user without the prior information of transmission power. In [3], an analytic model was developed using a two-dimensional Markov process for the performance analysis of an opportunistic spectrum sharing system. A queueing analytic framework was developed in [7] to study important performance measures for secondary users in a CR network.
In this paper ${ }^{1}$, we use techniques from signal detection theory and queueing theory to develop an adaptive spectrum mechanism for cognitive radio networks. We formulate spectrum detection as a hypothesis testing problem based on Bayes criterion to minimize average cost. The prior probabilities are estimated according to the maximum likelihood rule using a queueing theoretic model of the system occupancy. The spectrum detection scheme is extended to the case of multiple cooperating secondary users. Our numerical results show that the proposed adaptive spectrum detection scheme can perform much better than a static scheme with no knowledge of the prior probabilities, particularly under low primary traffic conditions. Cooperation among multiple secondary users further improves the error performance.

The remainder of the paper is organized as follows. Section II describes the system model and the problem formulation. Section III describes the Bayesian spectrum detection scheme. Section IV proposed a methodology for evaluating the prior probabilities for spectrum detection. Section V extends the spectrum detection mechanism to incorporate multiple cooperating secondary users. Section VI presents numerical results, illustrating the system performance with respect to the different metrics over a range of parameter settings. Finally, the paper is concluded in Section VII.

\section{System Model and Problem Formulation}

We consider a system of cognitive radios that are able to determine the spectrum occupancy over a band of frequency channels via signal strength (SS) measurements. For each frequency channel, a decision must be made on whether or not the channel is occupied by a primary user. Based on its perception of the spectrum occupancy, a secondary user may opportunistically make use of idle spectrum without causing harmful interference to the primary users.

Suppose the licensed spectrum band is divided into $N$ frequency channels serving the primary users. Secondary users detect the presence or absence of signals from primary users to maintain the records of the channel occupancy status. Spectrum detection is performed by a secondary user, or by a group of cooperating secondary users. Alternatively, the spectrum detection scheme may involve information exchange with an associated base station. The proposed model is applicable to both infrastructured and infrastructureless network architectures.

\footnotetext{
${ }^{1}$ This work was supported in part by the U.S. National Science Foundation under Grant CNS-0520151.
} 
In the proposed system model, a secondary user seeks to obtain an unused channel to service a secondary call. When a secondary user detects or is informed by others of the arrival of a primary call initiated by a primary user in its current channel, it immediately leaves the channel and switches to another unused channel, if one is available, to continue the call. If at that time all the channels are occupied, the secondary call will be lost. Primary users operates as if there are no secondary users in the service area. When a primary call arrives, it occupies a free channel if one is available; otherwise, the call is blocked.

In CR networks, the spectrum availability for the secondary users depends on the spectrum occupancy of the primary users. A distinct feature of such networks is that the secondary users have the capability to sense channel usage and switch between different channels, but must incur negligible interference on the primary users. Since the secondary users can cooperate with one another, we assume that they all have knowledge of the channel occupancies due to secondary users. Therefore, each secondary user must be able to decide, for each frequency channel, whether or not the channel is occupied by a primary user. We refer to this problem as the spectrum detection problem.

We propose a spectrum detection mechanism based on Bayes criterion via the SS measurements taken by the secondary users equipped with CR technology. The Bayes hypothesis testing problem is based on two assumptions. The first is that the source outputs are governed by prior probabilities. These probabilities represent the observer's information about the source before the decision action is made. The second assumption is that a cost is assigned to each possible course of action [8].

In the binary hypothesis testing, either hypothesis $H_{0}$ or $H_{1}$ is true. At the end of each decision interval one of four actions can be taken: 1) Choose $H_{0}$ when $H_{0}$ is true; 2) Choose $H_{1}$ when $H_{0}$ is true; 3) Choose $H_{1}$ when $H_{1}$ is true; and 4) Choose $H_{0}$ when $H_{1}$ is true. We denote the costs associated with the four courses of action as $C_{00}, C_{10}, C_{11}$, and $C_{01}$, respectively. The first subscript indicates the hypothesis chosen and the second indicates that the hypothesis is true. A decision rule should be designed to minimize the average cost. Typically, the cost associated with each action is assigned by the designer in accordance with the specific application. The prior probabilities, denoted by $P\left(H_{0}\right)$ and $P\left(H_{1}\right)$, are derived based on the observer's information. Hence, as part of the spectrum detection problem, we must address the following issue: Given observations over a given time interval, how can the prior probabilities be estimated in order to solve the spectrum detection problem?

\section{Spectrum Detection}

In this section, we develop the spectrum detection mechanism based on Bayes criterion, and then solve for the prior probabilities using queueing-theoretic techniques. We assume that a secondary user periodically scans the whole spectrum and takes signal strength (SS) measurements on channel $j, 1 \leq j \leq N$. We also assume that a secondary user knows the channel occupancy information of other secondary users, because the secondary users can exchange information among themselves, although they cannot communicate with primary users. Therefore, the decision that must be made concerning a given channel is whether the channel is idle or occupied by a primary user. The associated hypothesis testing problem for a given channel $j$ is formulated as follows:

$$
\begin{aligned}
& H_{0}: r_{j}=n_{j}, \\
& H_{1}: r_{j}=A_{j}+n_{j},
\end{aligned}
$$

where $r_{j}$ is the received signal strength on channel $j$ in $\mathrm{dB} ; n_{j}$ is the shadowing noise, modeled as a zeromean Gaussian random variable with variance $\sigma^{2}$; and $A_{j}$ represents the received signal strength on channel $j$ due to the transmitted signal power and path loss.

To achieve the minimum average cost, we use the Bayes criterion and obtain the likelihood ratio $\Lambda\left(r_{j}\right)$ [8]:

$$
\Lambda\left(r_{j}\right) \triangleq \frac{P\left(r_{j} \mid H_{1}\right)}{P\left(r_{j} \mid H_{0}\right)} \underset{H_{0}}{\stackrel{H_{1}}{\gtrless}} \frac{P\left(H_{0}\right)\left(C_{10}-C_{00}\right)}{P\left(H_{1}\right)\left(C_{01}-C_{11}\right)},
$$

where $P\left(H_{0}\right)$ represents the a priori probability that channel $j$ is idle, $P\left(H_{1}\right)$ is the a priori probability that channel $j$ is being used by a primary user. The conditional probability density of $r_{j}$ under each hypothesis follows easily:

$$
\begin{aligned}
& P\left(r_{j} \mid H_{0}\right)=\frac{1}{\sqrt{2 \pi} \sigma} \exp \left(-\frac{r_{j}^{2}}{2 \sigma^{2}}\right), \\
& P\left(r_{j} \mid H_{1}\right)=\frac{1}{\sqrt{2 \pi} \sigma} \exp \left(-\frac{\left(r_{j}-A_{j}\right)^{2}}{2 \sigma^{2}}\right) .
\end{aligned}
$$

Substituting into (1) and taking the logarithm, we obtain

$$
r_{j} \underset{H_{0}}{\stackrel{H_{1}}{\gtrless}} \frac{\sigma^{2}}{A_{j}}\left(\ln \frac{P\left(H_{0}\right)\left(C_{10}-C_{00}\right)}{P\left(H_{1}\right)\left(C_{01}-C_{11}\right)}+\frac{A_{j}^{2}}{2 \sigma^{2}}\right) \triangleq \gamma_{0} .
$$

Equation (2) provides a decision threshold. The secondary user simply measures the received SS on channel $j$, compares it with the threshold $\gamma_{0}$, and then makes a decision concerning the status of the channel.

In a practical system, the detection performance may be degraded by the presence of shadow fading and noise. A secondary user may incorrectly determine that a primary user is present in a channel when the channel is in fact idle. Alternatively, a secondary user may incorrectly determine that a channel is idle when in fact, the channel is being used by a primary user. The former event is referred to as a false alarm, and the latter is a misdetection. The misdetection event usually causes more harmful impact on the system performance than the false alarm event, because a misdtection may cause harmful interference to a primary user.

The false alarm probability, denoted by $p_{f}$, and misdetection probability, denoted by $p_{m}$, are two performance 
parameters for evaluating the above hypothesis testing problem. By definition, the two performance parameters can be calculated as follows:

$$
\begin{aligned}
p_{f} & =\int_{\gamma_{0}}^{\infty} \frac{1}{\sqrt{2 \pi} \sigma} e^{-\frac{r_{j}^{2}}{2 \sigma^{2}} d r_{j}=Q\left(\frac{\gamma_{0}}{\sigma}\right)} \\
p_{m} & =\int_{-\infty}^{\gamma_{0}} \frac{1}{\sqrt{2 \pi} \sigma} e^{-\frac{\left(r_{j}-A_{j}\right)^{2}}{2 \sigma^{2}}} d r_{j}=Q\left(\frac{A_{j}-\gamma_{0}}{\sigma}\right) .
\end{aligned}
$$

The average decision cost, denoted by $\mathcal{C}$, can be determined as

$$
\begin{aligned}
\mathcal{C} & =P\left(H_{0}\right)\left(C_{10} p_{f}+C_{00}\left(1-p_{f}\right)\right) \\
& +P\left(H_{1}\right)\left(C_{01} p_{m}+C_{11}\left(1-p_{m}\right)\right)
\end{aligned}
$$

If we assume that there is no cost for a correct decision and the cost for wrong decision is equal to 1 , i.e., $C_{00}=$ $C_{11}=0$ and $C_{01}=C_{10}=1$, then the cost $\mathcal{C}$ in (5) becomes the average error probability $P_{e}$, defined by

$$
P_{e}=P\left(H_{0}\right) p_{f}+P\left(H_{1}\right) p_{m} .
$$

\section{Estimation of PRIOR PROBABILITIES}

In this section, we formulate a system performance model to estimate the probabilities $P\left(H_{0}\right)$ and $P\left(H_{1}\right)$. In many applications, it is difficult to determine the prior probabilities for a hypothesis testing problem accurately. By observing the traffic dynamics of the primary users and secondary users during a certain time period, we can obtain estimate the prior probabilities, which can capture the dynamic characteristics of the traffic. This will ensure that the decision threshold will adapt according to the traffic dynamics. We will show that an adaptive spectrum detection mechanism can achieve much better performance compared to a static scheme that does not utilize prior knowledge, i.e., $P\left(H_{0}\right)=P\left(H_{1}\right)=0.5$ regardless of the traffic dynamics.

We shall assume that the traffic arrival process of each type of user is Poisson with rate $\lambda_{1}$ for primary calls and $\lambda_{2}$ for secondary calls. The service time for each type of users is exponentially distributed with parameter $\mu_{1}$ for the primary calls and $\mu_{2}$ for the secondary calls. Similar assumptions have been widely used to model call arrivals in wireless networks, e.g., [9], [10].

We first estimate the parameter $\mu_{1}$. Since a secondary user equipped with a cognitive radio can monitor the service time $T$ (i.e., the difference between the arrival time and departure time) of a primary user at a channel, and the service time is assumed to be exponentially distributed with rate $\mu_{1}$, when the secondary user observes a total of $L$ independent time samples in a certain time period, $T_{1}, T_{2}, \cdots, T_{L}$, the departure rate $\mu_{1}$ can be easily estimated through the likelihood function for this problem:

$$
f_{T_{1}, \cdots, T_{L} ; \mu_{1}}\left(T_{1}, \cdots, T_{L} ; \mu_{1}\right)=\prod_{i=1}^{L} \mu_{1} \exp \left(-\mu_{1} T_{i}\right) .
$$

By using the maximum likelihood estimation method [8], the maximum likelihood estimate (MLE) of $\mu_{1}$ is obtained as

$$
\hat{\mu}_{1}=\frac{1}{\frac{1}{L} \sum_{i=1}^{L} T_{i}} .
$$

Since the arrival process of the primary users is Poisson with rate $\lambda_{1}$, the interarrival time of a primary call can be derived to be exponentially distributed with parameter $\lambda_{1}$. Hence, using the same procedure, the estimate of $\lambda_{1}$ can be obtained. Similarly, estimates of $\lambda_{2}$ and $\mu_{2}$ are obtained.

Let $X_{1}(t)$ and $X_{2}(t)$ denote the number of primary and secondary calls using the channels at time $t$, respectively. The process $\left(X_{1}(t), X_{2}(t)\right)$ is a two-dimensional Markov process with state space $\mathcal{S}=\{(i, j) \mid 0 \leq i+j \leq$ $N\}$. The the transition rate diagram is for $\left(X_{1}(t), X_{2}(t)\right)$ is depicted in Fig. 1 (a) with $0 \leq i+j<N$ and in Fig. 1 (b) with $i+j=N$. Let $\pi(i, j)$ denote the steadystate probability that the system is in state $(i, j)$. The system balance equations can then be written as follows:

$$
\begin{array}{r}
\left(\lambda_{1}+\lambda_{2}+i \mu_{1}+j \mu_{2}\right) \pi_{i, j}=\lambda_{1} \pi_{i-1, j}+\lambda_{2} \pi_{i, j-1}+ \\
(i+1) \mu_{1} \pi_{i+1, j}+(j+1) \mu_{2} \pi_{i, j+1}, 0 \leq i+j<N \\
\left(\lambda_{1}+i \mu_{1}+j \mu_{2}\right) \pi_{i, j}=\lambda_{1} \pi_{i-1, j}+\lambda_{1} \pi_{i-1, j+1}+ \\
\lambda_{2} \pi_{i, j-1}, i+j=N, 0 \leq i \leq N-1 \\
N \mu_{1} \pi_{N, 0}=\lambda_{1} \pi_{N-1,0}+\lambda_{1} \pi_{N-1,1}, \quad i=N, j=0 .
\end{array}
$$

The above equations contain $(N+1)(N+2) / 2$ unknowns, i.e., the probabilities $\pi_{i, j}, 0 \leq i \leq N, 0 \leq$ $j \leq N-i$. But there are only $N(N+1) / 2$ independent equations in the above equations. Thus, $N+1$ more equations are needed. Recall that in our model, a primary call operates as if there is no secondary call in the service area. Hence, the probabilities $\pi_{i, 0}, 0 \leq i \leq N$, are given by the $\mathrm{M} / \mathrm{M} / \mathrm{N} / \mathrm{N}$ formula, i.e.,

$$
\pi_{i, 0}=\pi_{0,0} \frac{\left(\lambda_{1} / \mu_{1}\right)^{i}}{i !}, \quad 1 \leq i \leq N .
$$

The final equation is provided by the normalization condition:

$$
\sum_{i=0}^{N} \sum_{j=0}^{N-i} \pi_{i, j}=1
$$

Equations (8)-(10) are sufficient to evaluate the state probabilities $\pi_{i, j}$. After obtaining the state probabilities, we can evaluate the mean number of primary calls and secondary calls, denoted by $N_{1}$ and $N_{2}$, respectively, in the system under steady state:

$$
N_{1}=\sum_{i=1}^{N} \sum_{j=0}^{N-i} i \pi_{i, j}, \quad N_{2}=\sum_{i=0}^{N-1} \sum_{j=1}^{N-i} j \pi_{i, j} .
$$

From $N_{1}$ and $N_{2}$, we can solve for the mean probability of a channel being occupied by a primary call and the mean probability of a channel being idle, which are 


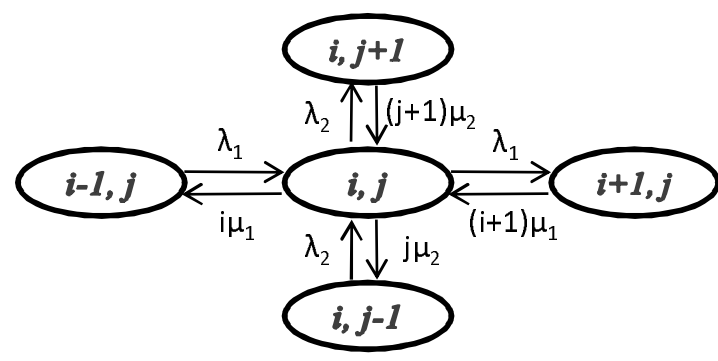

(a) $0 \leq \mathrm{i}+\mathrm{j}<\mathrm{N}$

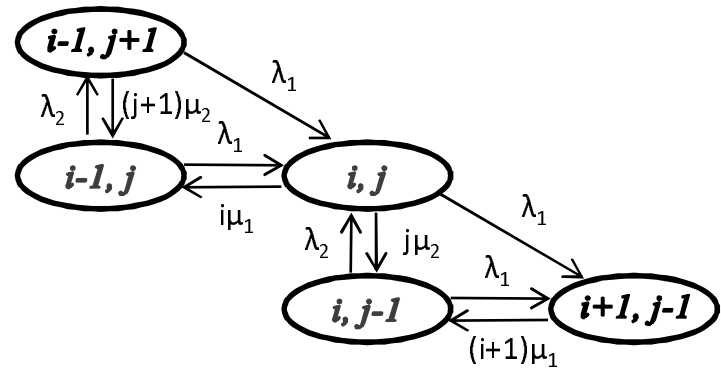

(b) $\mathrm{i}+\mathrm{j}=\mathrm{N}$

Fig. 1. State transition diagrams for the Markov process $\left(X_{1}(t), X_{2}(t)\right)$.

used to approximate the prior probabilities $P\left(H_{1}\right)$ and $P\left(H_{0}\right)$, respectively. Thus, we have

$$
P\left(H_{1}\right) \approx \frac{N_{1}}{N}, \quad P\left(H_{0}\right) \approx 1-\frac{N_{1}+N_{2}}{N} .
$$

Note that the above expressions for $P\left(H_{1}\right)$ and $P\left(H_{0}\right)$ will change according to the traffic load. Hence, the proposed spectrum detection mechanism, utilizing these probabilities, adapts to the dynamic traffic conditions. For example, when the primary traffic load increases, according to the above equations, we obtain a lower detection threshold, and hence the misdetection probability decreases.

\section{Cooperative Spectrum Detection}

In Section III, the hypothesis tests are conducted by a single secondary user, independent of other secondary users. Now we consider the hypothesis tests under collaboration among multiple secondary users. A secondary user and some of its neighbors synchronously measure the SS for a channel over a specific time interval and exchange information with each other or send results to the base station depending on different applications. Clearly, these measurements can be treated independently. Assume that $M$ users cooperate to measure the SS in channel $j$ during a certain time period. The secondary node making the decision obtains the $M$ independent measurement samples, calculates the average value, and then compares it with the detection threshold in order to make a decision. Since the measurements taken by different secondary users are independent Gaussian random variables, the average of these variables is a Gaussian variable $\bar{r}_{j}$ with variance given by

$$
\operatorname{Var}\left(\bar{r}_{j}\right)=\operatorname{Var}\left(\frac{1}{M} \sum_{i=1}^{M} r_{j i}\right)=\frac{\sigma^{2}}{M} .
$$

The mean of $\bar{r}_{j}$ is zero when channel $j$ is idle and $A_{j}$ when channel $j$ is being used by a primary user. Hence, the likelihood function under each hypothesis can be written as

$$
\begin{aligned}
& P\left(\bar{r}_{j} \mid H_{0}\right)=\left(\frac{M}{2 \pi \sigma^{2}}\right)^{\frac{1}{2}} \exp \left(-\frac{M \bar{r}_{j}^{2}}{2 \sigma^{2}}\right), \\
& P\left(\bar{r}_{j} \mid H_{1}\right)=\left(\frac{M}{2 \pi \sigma^{2}}\right)^{\frac{1}{2}} \exp \left(-\frac{M\left(\bar{r}_{j}-A_{j}\right)^{2}}{2 \sigma^{2}}\right) .
\end{aligned}
$$

The two types of error probabilities, i.e., false alarm probability $p_{f}^{\prime}$ and misdetection probability $p_{m}^{\prime}$ can be calculated as ${ }^{2}$ :

$$
\begin{aligned}
& p_{f}^{\prime}=\int_{\gamma_{0}}^{\infty} P\left(\bar{r}_{j} \mid H_{0}\right) d \bar{r}_{j}=Q\left(\frac{\sqrt{M} \gamma_{0}}{\sigma}\right) \\
& p_{m}^{\prime}=\int_{-\infty}^{\gamma_{0}} P\left(\bar{r}_{j} \mid H_{1}\right) d \bar{r}_{j}=Q\left(\frac{\sqrt{M}\left(A_{j}-\gamma_{0}\right)}{\sigma}\right) .
\end{aligned}
$$

Clearly, both types of error probabilities are reduced under cooperative detection, $p_{f}^{\prime}<p_{f}, p_{m}^{\prime}<p_{m}$, and the average cost is thus reduced.

\section{Numerical Results}

In this section, we present numerical results for the system model in terms of the prior probabilities $P\left(H_{1}\right)$ and $P\left(H_{0}\right)$, the mean error probability of detection $P_{e}$, and the two types of error probabilities $p_{f}$ and $p_{m}$. The system parameters are set as follows ${ }^{3}: N=12, M=5$, $A_{j}=3, \sigma=1, \mu_{1}=20, \mu_{2}=20, \lambda_{2}=60$ or 80 , and $\lambda_{1}$ ranges from 10 to 100 .

Fig. 2 shows how the prior probabilities vary as functions of the traffic intensities for both types of traffic. As should be expected, when the primary call arrival rate $\lambda_{1}$ increases, the probability that the channel is occupied by primary users, i.e., the probability $P\left(H_{1}\right)$, increases, while the probability that the channel is idle, i.e., $P\left(H_{0}\right)$, decreases. As $\lambda_{2}$ increases, $P\left(H_{0}\right)$ decreases, but $P\left(H_{1}\right)$ does not change. This is because $P\left(H_{1}\right)$ depends only on the channel occupancy of primary calls and is independent of the behavior of the secondary calls.

Fig. 3 compares the mean error probability $P_{e}$ under the proposed adaptive spectrum detection scheme with the case where the prior probabilities are assumed to have the values $P\left(H_{0}\right)=P\left(H_{1}\right)=0.5$. We observe that the proposed scheme can achieve much better performance, especially when the primary traffic load is relatively light. We also observe that in the

\footnotetext{
${ }^{2}$ For the purpose of comparison, here we assume that the detection threshold $\gamma_{0}$ is the same under both single user and multiple user detection.

${ }^{3}$ Time units are assumed to be dimensionless, but can easily be mapped to standard units.
} 


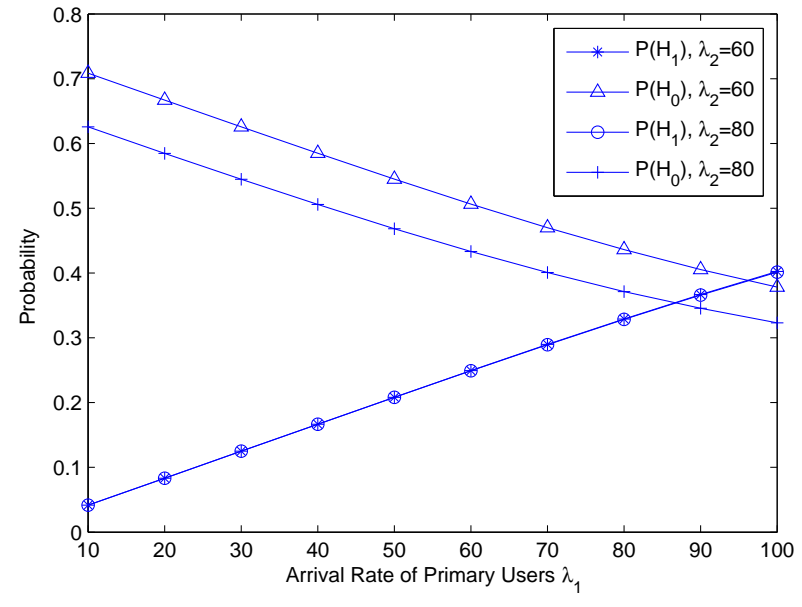

Fig. 2. Mean channel occupancy and idle probabilities $P\left(H_{1}\right)$ and $P\left(H_{0}\right)$.

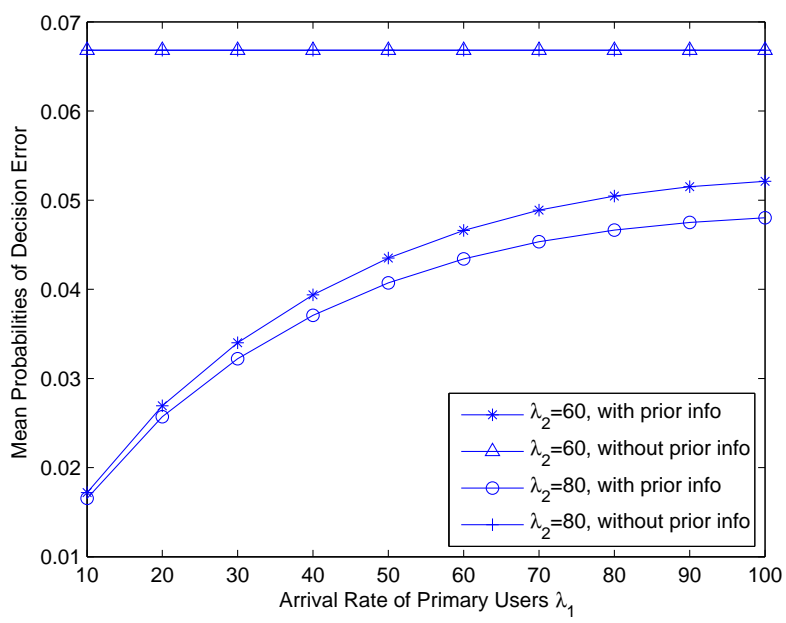

Fig. 3. Mean error probability $P_{e}$ under various scenarios.

proposed scheme, as $\lambda_{2}$ increases, $P_{e}$ decreases. This is because we assume that secondary users are mutually cognizant of the channels that they occupy since they can exchange information among themselves. As more secondary calls occupy channels, the size of the decision space decreases, leading to a smaller value of $P_{e}$.

Fig. 4 evaluates the two types error probabilities under the multiple user detection scheme discussed in Section $\mathrm{V}$. We observe that both types of error probabilities, i.e., $p_{f}$ and $p_{m}$, are reduced under cooperative detection with multiple users.

\section{CONCLUSION}

We proposed an adaptive spectrum detection mechanism for cognitive radio networks in dynamic traffic environments. The spectrum detection mechanism is formulated as a hypothesis testing problem and developed based on Bayes criterion to minimize average cost. To determine the prior probabilities used for spectrum detection, we estimate the traffic dynamics of both types of users via the maximum likelihood rule. The traffic

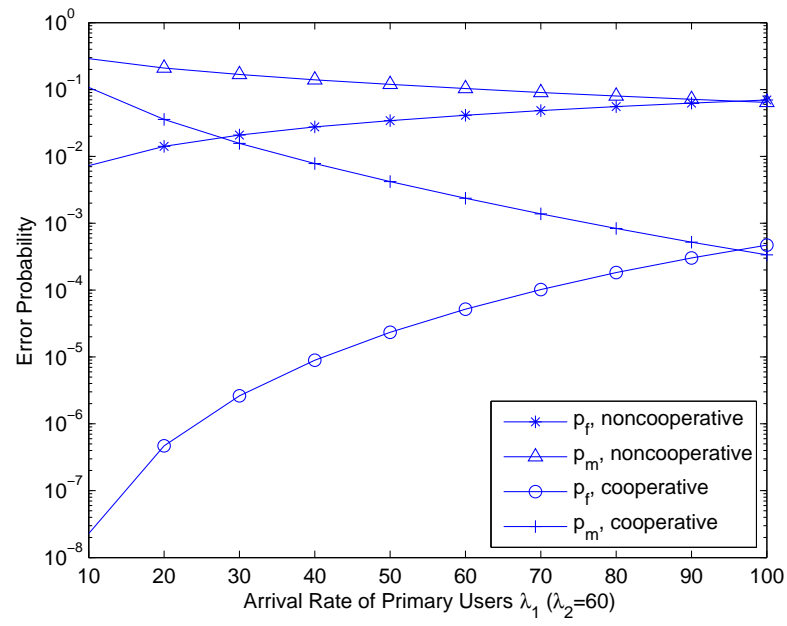

Fig. 4. Comparison of the two types of error probabilities.

estimates are applied to a two-dimensional Markov process model of the system occupancy to evaluate the prior probabilities. We also extended the scheme to leverage cooperation among multiple secondary users. Our numerical results suggest that the proposed adaptive spectrum detection scheme can achieve much better performance compared to the case where no knowledge of prior probabilities is available, especially under light primary traffic loads. The results also indicate the benefits of cooperative spectrum detection in terms of reducing both the false alarm and misdetection probabilities.

\section{REFERENCES}

[1] M. McHenry, "Frequency agile spectrum access technologies," in Proc. FCC Workshop on Cognitive Radio, May 2003.

[2] S. Haykin, "Cognitive radio: Brain-empowered wireless communications," IEEE J. Selected Areas in Comm., vol. 23, pp. 201220, Feb. 2005.

[3] S. Tang and B. L. Mark, "Performance analysis of a wireless network with opportunistic spectrum sharing," in Proc. IEEE Globecom'07, pp. 4636-4640, Nov. 2007.

[4] S. Geirhofer, L. Tong, and B. Sadler, "A measurement-based model for dynamic spectrum access in WLAN channels," in Proc. IEEE Military Commun. Conf. (Milcom), pp. 1-7, Oct. 2006.

[5] X. Liu and S. Shankar N., "Sensing-based opportunistic channel access," Mobile Networks and Applications, vol. 11, pp. 577-591, Aug. 2006.

[6] S. Kim, H. Jeon, and J. Ma, "Robust localization with unknown transmission power for cognitive radio," in Proc. IEEE Military Commun. Conf. (Milcom), (Orlando, FL, USA), Oct. 2007.

[7] M. Rashid, J. Hossain, E. Hossain, and V. Bhargava, "Opportunistic spectrum access in cognitive radio networks: A queueing analytic model and admission controller design," in Proc. IEEE Globecom'07, pp. 4647-4652, Nov. 2007.

[8] H. L. Van Trees, Detection, Estimation and Modulation Theory: Part I. New York: John Wiley \& Sons, Inc., paperback ed., 2001.

[9] H. Wu, Y. Wang, H. Dang, and F. Lin, "Analytic, simulation, and empirical evaluation of delay/fault-tolerant mobile sensor networks," IEEE Trans. on Wireless Comm., vol. 6, pp. 32873296, Sep. 2007.

[10] B. Hamdaoui and P. Ramanathan, "A cross-layer admission control framework for wireless ad-hoc networks using multiple antennas," IEEE Trans. on Wireless Comm., vol. 6, pp. 40144024, Nov. 2007. 